\title{
Antioxidant properties of benzoic acid derivatives against superoxide radical
}

\author{
Beata Velika,* Ivan Kron \\ Doc. Ing. Ivan Kron, CSc.Department of Chemistry, Biochemistry, Clinical Biochemistry and LABMED a.s., \\ Faculty of Medicine, University of Pavol Jozef Safarik in Kosice, Slovakia \\ RNDr. Beata Velika, phD., Department of Chemistry, Biochemistry, Clinical Biochemistry and LABMED a.s., \\ Faculty of Medicine, University of Pavol Jozef Safarik in Kosice, Slovakia
}

Submission Date: 27-10-2012; Accepted Date: 8-11-2012

\begin{abstract}
Introduction: Reactive species play a dual deleterious and beneficial antioxidant role. Overproduction of reactive species can cause damage of cell structures, including lipids and membranes, proteins and DNA. Hydroxybenzoic acid belongs to a group of phenolic compounds. Hydroxybenzoic acids itself and its derivatives showed antioxidant properties against different type of free radicals and can prevent or decrease overproduction of reactive species. Methods: In this study we tested antioxidant properties of 14 different benzoic acid derivatives against superoxide radical. The antioxidant properties of tested compound were determined by Beauchamp and Fridovich methods (1971). We studied kinetics and relationship between structure and antioxidant activity of the compounds. Results: The monohydroxybenzoic derivatives showed the best antioxidant properties. The results showed different antioxidant properties of hydroxybenzoic acid derivatives in 5, 10, 15 and 20 minutes of the reaction. Very important is the position of - $\mathrm{OH}$ group to carboxylate group. The compounds with hydroxyl group in ortho and para position to the carboxylate group showed the best antioxidant properties. Derivatives with blocked - $\mathrm{OH}$ group showed very low antioxidant properties in comparison with derivatives with blocked $-\mathrm{COOH}$ group. Conclusion: The structure and position of hydroxyl group is very important for antioxidant acitivy.
\end{abstract}

Keywords: Antioxidant, free radical, hydroxybenzoic acid, superoxide radical.

\section{INTRODUCTION}

Reactive oxygen species (ROS), are products of normal cellular metabolism. ROS can be either harmful or beneficial to living system. ${ }^{[1]}$ The production of superoxide occurs mostly within the mitochondria of the cell. ${ }^{[2]}$ The mitochondrial electron transport chain is the main source of ATP in the mammalian cell and thus is essential for life. During energy transduction, a small number of electrons

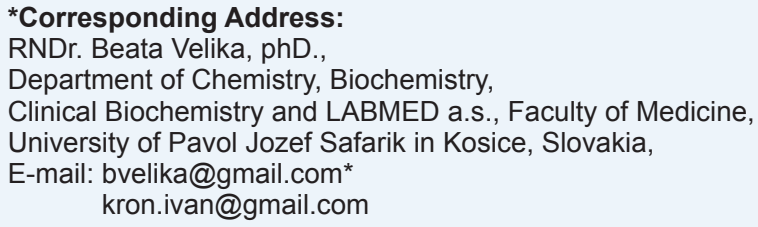

DOI: $10.5530 / a x .2012 .4 .11$ leaks to oxygen prematurely, forming the oxygen free radical superoxide, which has been implicated in pathophysiology of variety diseases. ${ }^{[3]}$ In healthy subjects, it has been estimated that approximately $1.7-17 \mathrm{~kg}$ per year of dioxygen is converted to $\mathrm{O}^{2--}$ and hence this is likely the most abundant oxygen radical in the human body. ${ }^{[4]}$ This radical is believed to be responsible for $\mathrm{O}_{2}$ toxicity in living organisms.

Phenolic compounds are well known as antioxidants against superoxide radical. Phenolic compounds and some of their derivatives are very efficient in preventing autooxidation, however, only a few phenolic compounds are currently permissible by law as food antioxidant. ${ }^{[5]}$ Phenols are known to reduce the rates of oxidation of organic mater by transferring a $\mathrm{H}$ atom (from their $\mathrm{OH}$ group) to the chain-carrying ROO radicals. ${ }^{[6]}$ Salicylates belongs 
Beata Velika, et al.: Antioxidant properties of benzoic acid derivatives against superoxide radical

to the group of phenolic compounds. Salicylic acid (2-hydroxybenzoic acid) is one of the oldest drug used in medicine, either free or in the ester form with acetic acid. ${ }^{[7]}$ Besides antipyretic, antirheumatic and analgesic properties it shows also antioxidant activity due to the presence of a phenol group. 4-hydroxybenzoic acid is primarily known as the basis for the preparation of its esters, known as parabens. Parabens are a group of chemicals widely used as preservatives in the cosmetic and pharmaceutical industries. The determination of the antioxidant capacity of 4-hydroxybenzoic acid esters (parabens) against superoxide radical is very important because of the frequent use of parabens in cosmetics. These compounds are used for their bacteriocidal and fungicidal properties, also as food additives. Their concentration in cosmetic are usually $0.3-1 \%$, about two orders of magnitude higher than in the foods in which the compounds are permitted for use. ${ }^{[8,9,10]}$ When parabens are administered orally, they are readily hydrolyzed to 4-hydroxybenzoic acid, metabolized to conjugates of glucuronic acid, sulfuric acid and glycine in the liver and kidneys and then excreted in the urine. ${ }^{[8,9,10]}$ For this reason, parabens are generally considered as non toxic compounds. The effect of other hydroxy group position could be important for increasing antioxidant activity. Kinetic analysis showed significant differences between the derivates with the best antioxidant properties recorded for the 2-hydroxybenzoic acid, which is in accordance for antioxidant activities determined with TROLOX. ${ }^{[1]}$

The authors discuss the structure-antioxidant activity relationship related to kinetic of the electron transfer within molecules of hydroxybenzoic acids. This substances should be used as a model compounds for the study of antioxidant activity of phenolic compounds, because of their variability, solubulity, accessibility, inexpensivity. These compounds are constituents of natural antioxidants as chalcones, flavones, flavanones.

The main aim of our work was to determine relationship between structure and antioxidant activity of unsubstituted and substituted hydroxybenzoic acids on the dependence of the localization of hydroxyl substituent against carboxyl group.

\section{MATERIAL AND METHODS}

\section{Chemicals}

All used chemicals were of the highest quality available. 2-hydroxybenzoic acid (salicylic acid, SA), 3-hydroxybenzoic acid (3-HBA), 4-hydroxybenzoic acid (4-HBA), 2,4-dihydroxybenzoic acid (2,4-DHB), 2,6-dihydroxybenzoic acid
(2,6-DHB), 3,5-dihydroxybenzoic acid (3,5-DHB), acetylsalicylic acid (ASA), L-metihionine and Nitro blue tetrazolium (NBT) were purchased from MERCK (Merk Schuchardt OHG, Hohenbrunn, Germany). 2,3-dihydroxybenzoic acid (2,3-DHB), 2,5-dihydroxybenzoic acid (2,5-DHB), 3,4-dihydroxybenzoic acid (3,4-DHB), caffeic acid (CA), gallic acid (GA), 4-hydroxybenzoic acid propyl ester (4-HBAP) and 4-hydroxybenzoic acid methyl ester (4-HBAM) were purchased from SIGMA Inc. (St. Louis, MO, USA). $\mathrm{K}_{2} \mathrm{HPO}_{4}$ and $\mathrm{KH}_{2} \mathrm{PO}_{4}$ (ITES, Vranov nad Toplou), EDTA, $50 \mathrm{mM}$ phosphate buffer $\left(\mathrm{KH}_{2} \mathrm{PO}_{4}\right.$ and $\left.\mathrm{K}_{2} \mathrm{HPO}_{4}\right) \mathrm{pH} 7.4,13 \mathrm{mM}$ L-methionine, $0.1 \mathrm{mM}$ EDTA, $0.2 \mathrm{mM}$ riboflavin, $5 \mathrm{mM}$ NBT (nitro blue tetrazolium).

\section{The reaction mixture}

The reaction mixture contained $300 \mu \mathrm{l}$ of $0.2 \mathrm{mM}$ riboflavin, $30 \mu \mathrm{l}$ of $5 \mathrm{mM}$ NBT, $8.7 \mathrm{ml}$ of $50 \mathrm{mM}$ phosphate buffer $\mathrm{pH}$ 7.4, EDTA, L-methionine. The benzoic acid derivatives were added at the concentration $5 \mu \mathrm{g} / \mathrm{ml}$, $25 \mu \mathrm{g} / \mathrm{ml}, 50 \mu \mathrm{g} / \mathrm{ml}, 75 \mu \mathrm{g} / \mathrm{ml}$ and $100 \mu \mathrm{g} / \mathrm{ml}$. Riboflavin was added as last. The reaction was initiated by placing vessels under the $\mathrm{Hg}$ lamp. Illumination time was performed at $5 \mathrm{~min}, 10 \mathrm{~min}, 15 \mathrm{~min}$ and $20 \mathrm{~min}$ time points. The disturbing metal ions were removed by EDTA. The percentage of inhibition was calculated from the absorbances at 450 and $560 \mathrm{~nm}$ wavelengths.

\section{Antioxidant properties of hydroxybenzoic acid derivatives}

Antioxidant properties of hydroxybenzoic acid derivatives were measured by a test reaction according Beauchamp and Fridovich. ${ }^{[12]}$ After UV illumination was riboflavin reduced by L-methionine under aerobic conditions. The reduced form reacts with oxygen and formed a peroxide derivative, which was decomposed to superoxide radical anion. The superoxide ions were captured by the NBT (nitro blue tetrazolium) compound. The original yellow colour of NBT turns blue after the reduction. The reduction was determined by spectrophotometry method at $450 \mathrm{~nm}$ and $560 \mathrm{~nm}$ wavelengths. The presence of any antioxidant (captures the superoxide radical ion) inhibited photoreduction of NBT and the studied sample was decolorized.

\section{RESULTS}

The absorbance of the reagent solution at $450 \mathrm{~nm}$ and $560 \mathrm{~nm}$ in 5, 10, 15 and 20 minutes was measured after illumination. The percentage of inhibition of the compounds depended on concentration in different time points of the reaction (Fig. 1, Fig. 2, Fig. 3, Fig. 4, Fig. 5, Fig. 6, Fig. 7, Fig. 8). 
Beata Velika, et al.: Antioxidant properties of benzoic acid derivatives against superoxide radical

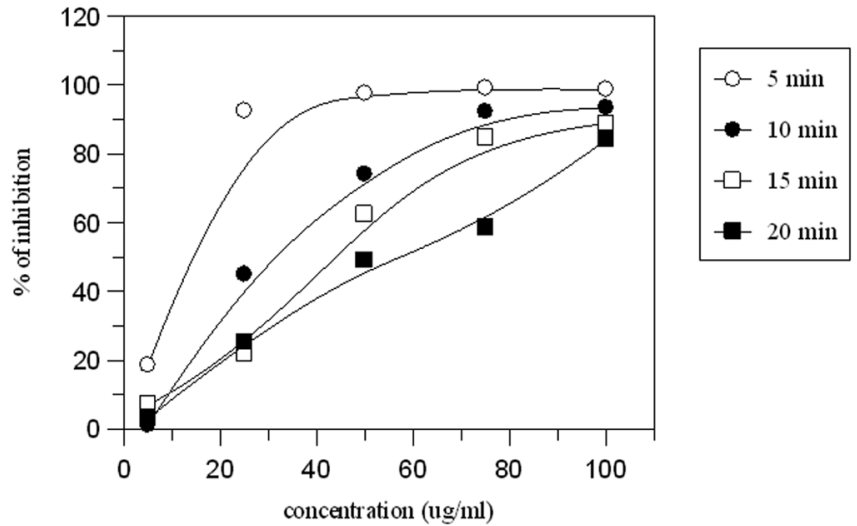

Figure 1. Time and concentration dependance of antioxidant activity of 2-HBA.
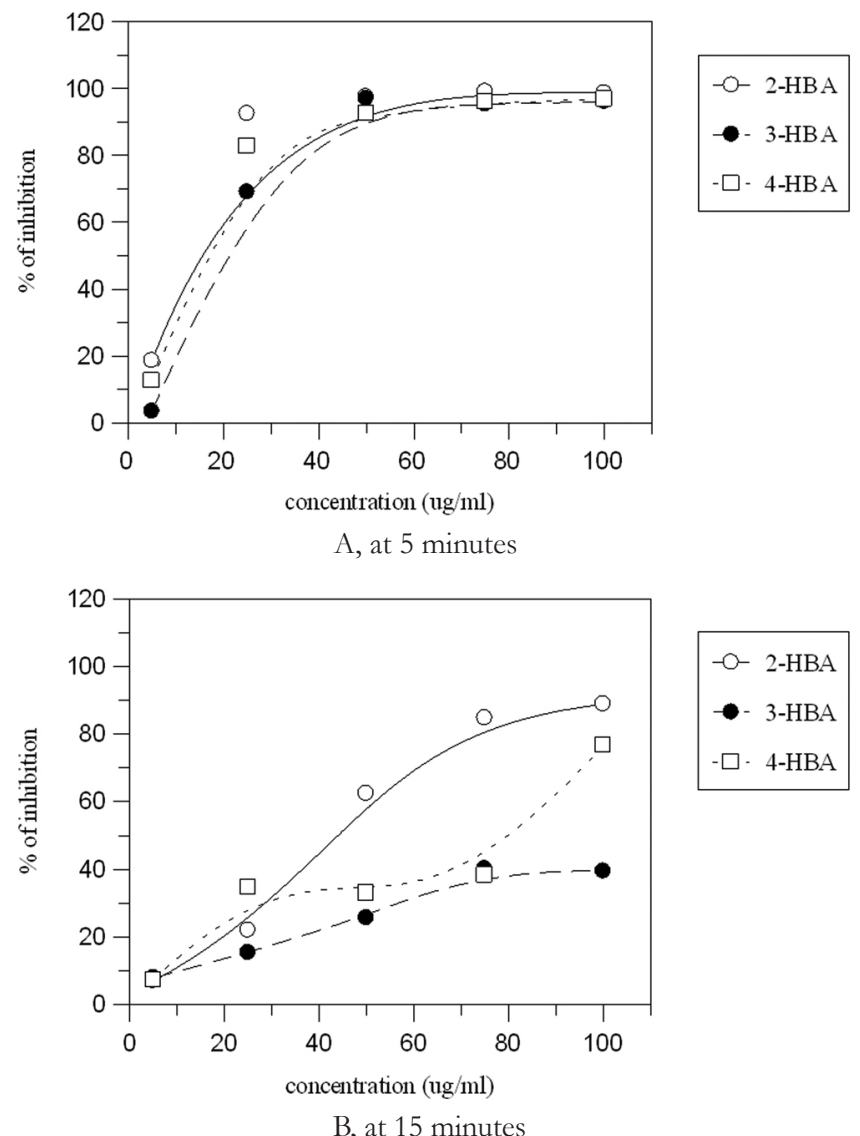

Figure 2. Concentration dependance of antioxidant activity of monohydroxybenzoic acids at 5 and 15 minutes.

The studied concentration range of tested compounds was from $25 \mu \mathrm{g} / \mathrm{ml}$ to $100 \mu \mathrm{g} / \mathrm{ml}$. The percentage of inhibition was calculated by using the formula:

$$
\% \text { of inhibition }=\left[\mathrm{A}_{0}(\text { time })-\mathrm{A}_{\mathrm{x}}(\text { time }) / \mathrm{A}_{0}(\mathrm{time})\right] \times 100
$$

where $A_{0}$ is the absorbance of the composition without antioxidant, and $A_{x}$ is absorbance of composition with antioxidant in the time.

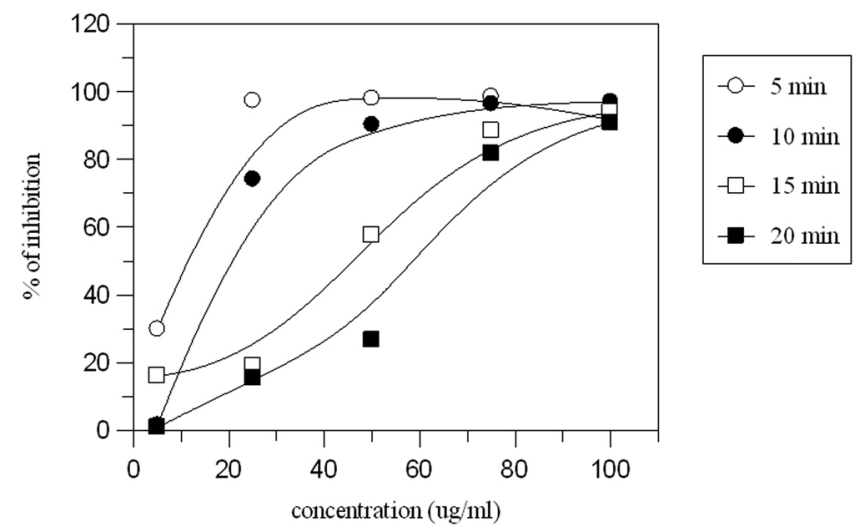

Figure 3. Time and concentration dependance of antioxidant activity of 2,5-DHB.
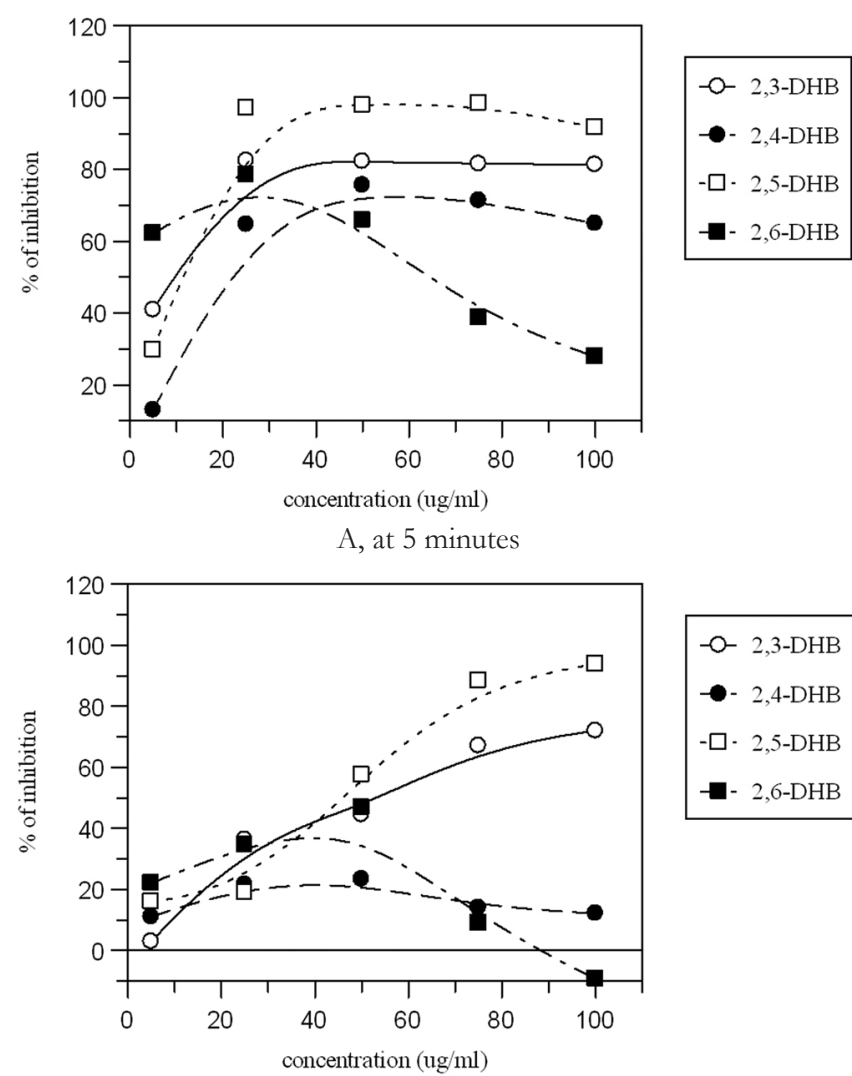

\begin{tabular}{|l|}
$-0-2,3-\mathrm{DHB}$ \\
$-2,4-\mathrm{DHB}$ \\
$-\square \cdot 2,5-\mathrm{DHB}$ \\
$-2,6-\mathrm{DHB}$
\end{tabular}

$\mathrm{B}$, at 15 minutes

Figure 4. Concentration dependance of antioxidant activity of dihydroxybenzoic acid at 5 and 15 minutes.

Antioxidant activity depends on the structures of hydroxybenzoic acid derivatives which was influenced by the presence, number position and interaction of the hydroxyl group on the hydroxybenzoic acid.

The monohydroxybenzoic acids showed higher antioxidant activity with hydroxy substituent placed in the ortho and para position in comparison with meta position in 
Beata Velika, et al.: Antioxidant properties of benzoic acid derivatives against superoxide radical

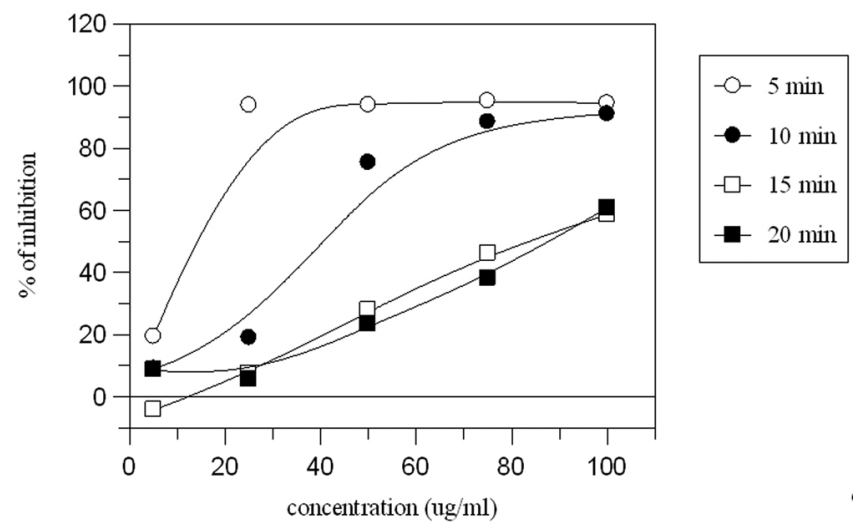

Figure 5. Time and concentration dependance of antioxidant activity of 3,4-DHB.

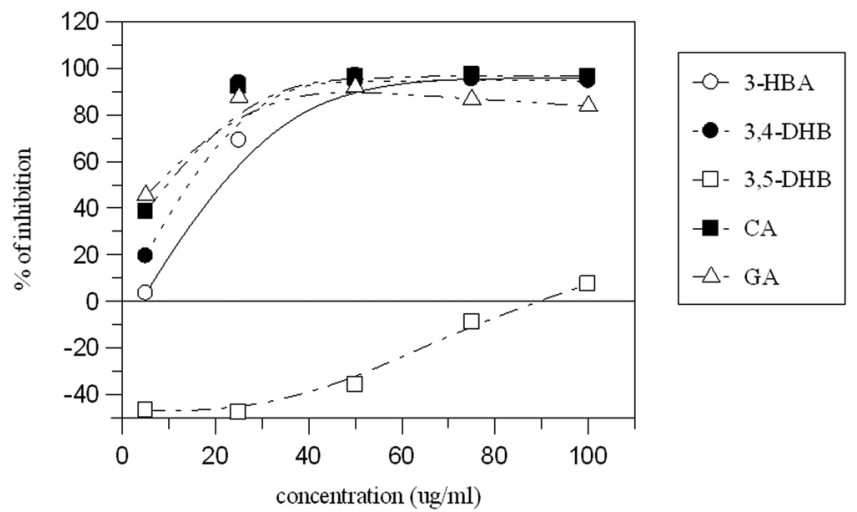

$\mathrm{A}$, at 5 minutes

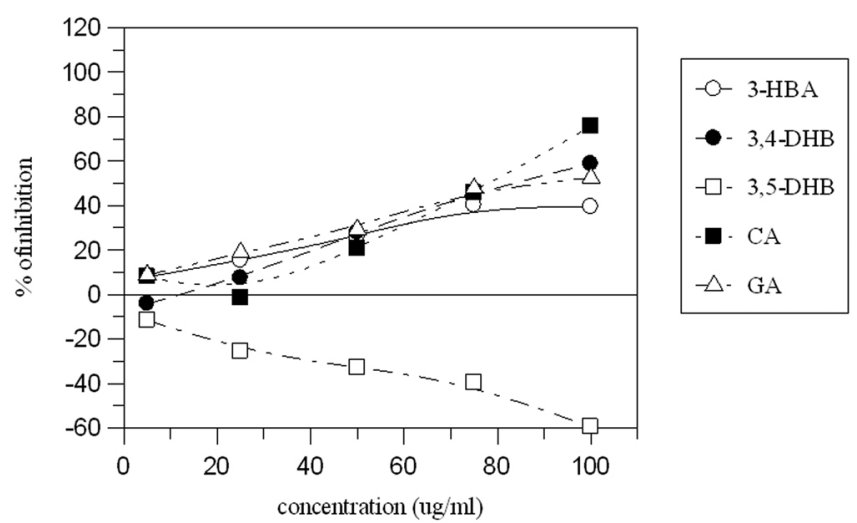

$\mathrm{B}$, at 15 minutes

Figure 6. Concentration dependance of antioxidant activity of 3 -HBA and its derivatives at 5 and 15 minutes.

terms of hydrogen-donating capacity against radical generated in the aqueous phase (Fig. 2A, 2B).

The higher antioxidant activity against superoxide radical showed dihydroxybenzoic acids after 5 minutes of the reaction. The best antioxidant properties showed 2,5-DHB and the the worst antioxidant properties from this group of tested compounds showed 2,6-DHB (Fig. 4A, 4B).

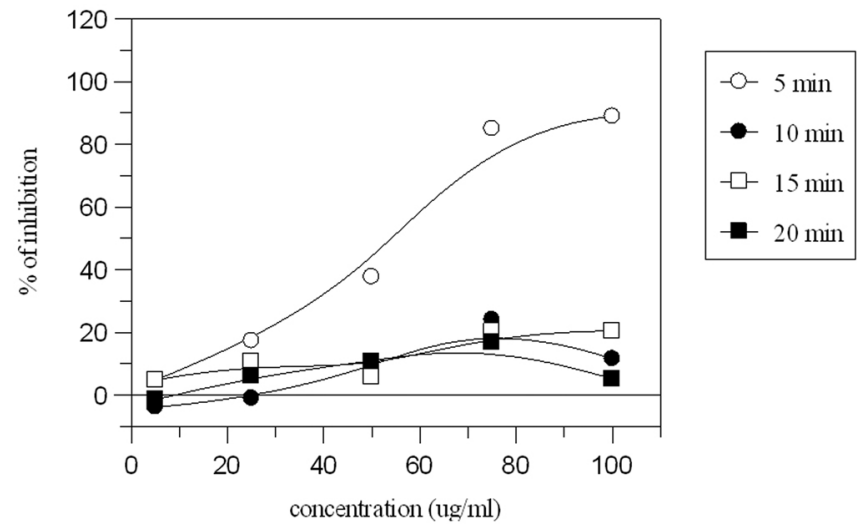

Figure 7. Time and concentration dependance of antioxidant activity 4-HBAM.
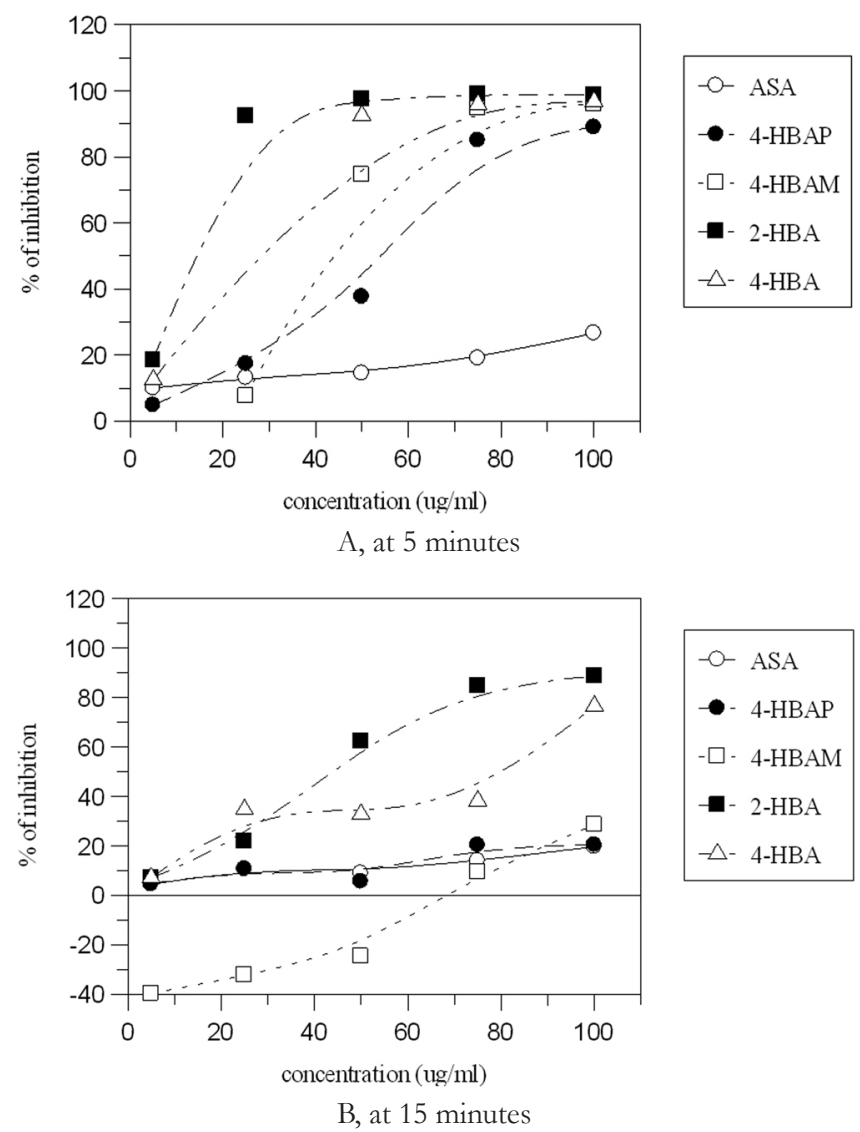

Figure 8. Concentration dependance of antioxidant activity of HBA esters at 5 and 15 minutes.

In the group of derivatives of 3-HBA, is very important localisation of hydroxyl groups, too. The comparison of antioxidant properties of 3-HBA, 3,4-DHB, 3,5-DHB, Caffeic acid and Gallic (Fig. 5, Fig. 6) in the $5 \mathrm{~min}$ a 15 min kinetic studies measurements time, all studied compounds showed better antioxidant properties after five minutes. 3,5-DHB showed prooxidant properties at the concentration 5, 25, 50 and $75 \mu \mathrm{g} / \mathrm{ml}$ (Fig. 6A, 6B). 
Beata Velika, et al.: Antioxidant properties of benzoic acid derivatives against superoxide radical

Table 1 Used compounds

\begin{tabular}{|c|c|c|c|c|}
\hline MHB & DHB & DHB & THB & esters \\
\hline \multirow[t]{4}{*}{ 2-HBA } & 2,3-DHB & & & ASA \\
\hline & 2,4-DHB & & & \\
\hline & $2,5-\mathrm{DHB}$ & & & \\
\hline & 2,6-DHB & & & \\
\hline \multirow{2}{*}{ 3-HBA } & 3,4-DHB & ${ }^{*}(-\mathrm{CH}=\mathrm{CH}-) \mathrm{CA}$ & $3,4,5-\mathrm{THB}(\mathrm{GA})$ & \\
\hline & 3,5-DHB & & & \\
\hline \multirow[t]{2}{*}{ 4-HBA } & & & & 4-HBAM \\
\hline & & & & 4-HBAP \\
\hline
\end{tabular}

The comparison of 4-hydroxybenzoic acid with its esters showed the highest antioxidant activity of 4-hydroxybenzoic acid. Propyl ester had lower antioxidant properties than the methyl ester of 4-hydroxybenzoic acid, probably as the result of the size of the characteristic group (Fig. 7, 8A, 8B).

\section{DISCUSION}

Phenols are presented as a variety group of compounds which are well known as antioxidants. One of them are benzoic acid derivatives, widely used as preservatives in food, cosmetic and pharmaceutical industry. Many various derivatives of benzoic acid are available therefore were chosen for model compounds on which the antioxidant properties were studied. Phenols or hydroxybenzoic acid derivatives are offten found in natural compounds like chalcones or generally flavonoids. Benzoic acid derivatives were tested for their ability to react with superoxide radicals generated by methionine-riboflavin generator. ${ }^{[12]}$ The antioxidant activity of phenolic acids and their derivatives depends on the number of hydroxyl groups in the molecule that would be stregthened by steric hindrance. ${ }^{[13]}$ The electron-withdrawing properties of the carboxylate group in benzoic acids has a negative influence on the H-donating of the hydroxybenzoic acid derivatives. ${ }^{[1]}$ Carboxyl group is an electron-withdrawing group, which does not benefit radical scavenging. However, the deprotonated carboxyl becomes an electron donating group which favors $\mathrm{H}$-atom-transfer and electron-donating based radical scavenging. ${ }^{[14]}$

Very important is time dependance of antioxidant activity of used compounds and the position of $-\mathrm{OH}$ group and $-\mathrm{COOH}$ group on benzene skelet.

The mohohydroxybenzoic acids (2-HBA, 3-HBA, 4-HBA) were studied in this paper. The best antioxidant properties in this group showed 2-HBA (Fig. 1). Antioxidant properties depended in the time. The monohydroxybenzoic acids showed higher antioxidant activity in the ortho and para position in comparisom with meta position (Fig. 2A, 2B) in terms of hydrogen-donating capacity against radical generated in the aqueous phase. The reason is position of hydroxyl group against carboxyl group. Antioxidant activity of all compounds with one hydroxyl group decreased in the time, mainly 3-HBA. The best antioxidant properties showed 2-HBA, but after first 5 minutes of the reaction all of used HBA showed very similar antioxidant properties against superoxide radical (Fig. 2A, 2B).

Antioxidant properties depends on the position of hydroxyl groups on benzene sceleton. 2,4-DHB and 2,6DHB showed antioxidant properties mainly after 15 and 15 minutes. The reason is the position of hydroxyl -OH group. In the case 2,5-DHB hydroxyl groups are in ortho and para position against carboxyl group, because of this conditions 2,5-DHB showed the best antioxidant properties. The worst antioxidant in this group is 2,6-DHB and 2,4-DHB because the second - $\mathrm{OH}$ group is in the meta position, what gives a negative effect on antioxidant properties of compounds (Fig. 3, Fig. 4A, 4B).

In the group of derivatives of 3-HBA, is very important localisation of hydroxyl groups, too. The comparison of antioxidant properties of 3-HBA, 3,4-DHB, 3,5-DHB, caffeic acid and gallic acid after 5 and 15 minutes, showed better antioxidant properties of all studied compounds after five minutes. Molecule 3,5-DHB showed the prooxidant properties at the concentration 5, 25, 50 and $75 \mu \mathrm{g} / \mathrm{ml}$. This compounds showed very low antioxidant properties at the concentration $100 \mu \mathrm{g} / \mathrm{ml}$. It is because of the position of the hydroxyl -OH groups on benzene skeleton is at meta position against carboxylate group, and each of hydroxyl groups is in meta position against each other. Compounds 3,4-DHB and caffeic acid showed very similar antioxidant properties, caused probably by the same position of hydroxyl substituents. There is difference of the structure, because of $-\mathrm{C}=\mathrm{C}$ occured in 
Beata Velika, et al.: Antioxidant properties of benzoic acid derivatives against superoxide radical

the structure of caffeic acid, but without any important effect. Gallic acid showed very good antioxidant properties, very similar as 3,4-DHB, it is because of ortho position of hydroxyl groups on benzene skelet (Fig. 5, Fig. 6A, 6B). Methylparabene, propylparabene as esters of 4-HBA and acetylsalicylic acid as ester of 2-HBA were tested. Blocked of $-\mathrm{COOH}$ group has a lower effect in compare with blocked -OH group (phenolic group). Acetylsalicylic acid with the blocked $-\mathrm{OH}$ group showed the lowest antioxidant properties against superoxide radical of all used esters and of all used compounds. 4-HBAM showed better antioxidant properties as 4-HBAP its because of shorter methyl group bounded on 4-HBA scelet. The comparison of 4-hydroxybenzoic acid with its esters, showed the highest antioxidant activity of 4-hydroxybenzoic acid. Propyl ester had lower antioxidant properties than the methyl ester of 4-hydroxybenzoic acid. This observation was caused by the size of the characteristic group (Fig. 7, Fig. 8 A, B).

Very important was the resonance effect caused by resonance structure of used compounds. These compounds are able to capture and to acquit an electrone what depends on the structure of used compound. It is signify as colorized and decolorized of NBT. This mechanism commemorate the system of the coenzyme Q in respiratory chain. This mechanism should explain the leaking of electrons from the respiratory chain - mainly coenzyme Q.

\section{CONCLUSIONS}

Antioxidant properties of all used compounds were measured at time 5, 10, 15 and 20 minutes. In this paper we used results after $5 \mathrm{~min}$ and 15 minutes, which are most significant. All these compounds showed the time dependance antioxidant activity with the highest activity after five minutes in compared with the results after fifteen ( or twenty) minutes. The intramolecular hydrogen bonding has important effects on the antioxidant activity of phenols. Various ortho-substituents may function as H-bond acceptors, however only the effects of methoxy and hydroxy groups will be described since these groups are largely diffused among natural phenols and have opposite effects on the antioxidant properties of phenols. ${ }^{[6]}$ The compounds with the best antioxidant properties are monohydroxybenzoic acids. The monohydroxybenzoic acids showed the best antioxidant activity in the ortho and para position, 2-HBA is better antioxidant as $4-\mathrm{HBA}$ and far better than $3-\mathrm{HBA}$. It is because of hydrogen-donating capacity against radicals generated in system. The compound with the best antioxidant properties from all tested compounds is 2-hydroxybenzoic acid (salicylic acid). The dihydroxybenzoic acids and its derivatives showed an antioxidant response dependent on the relative positions of the hydroxyl groups in the ring. Dihydroxybenzoic acids with the $-\mathrm{OH}$ groups in the ortho and para position to the carboxylate group showed better antioxidant properties against superoxide radical as dihydroxybenzoic acid with the $-\mathrm{OH}$ groups in the meta position to the carboxylate group. The worst antioxidant are 3,5-DHB and 2,6-DHB. This compounds showed prooxidant not antioxidant properties. Incorporateing of the $-\mathrm{CH}=\mathrm{CH}$ - between the phenyl ring and carboxylic group (in the case of caffeic acid) showed very small decreasing of the antioxidant activity of this compound in compare with 3,4-DHB. Gallic acid with three hydroxyl groups showed good antioxidant activity corresponding to localisation of this $-\mathrm{OH}$ groups on benezene ring. Gallic acid can efficiently scavenge free radicals. After deprotonisation it gives birth to gallic acid anion, which has big influence on radical scavenging behaviors.

\section{REFERENCES}

1. Valko M, Rhodes CJ, Moncol J, Izakovic M, Mazur M. Free radicals, metals and antioxidants in oxidative stress-induced cancer. Chem. Biol. Interact 2006; 160:1-4.

2. Cadenas E, Sies H. The lag phase. Free Radic. Res 1998; 28:601-9.

3. Kovacic P, Pozos RS, Somanathan R, Shangari N, O'Brien PJ. Mechanism of mitochondrial uncouplers, inhibitors, and toxins: Focus on electron transfer, free radicals, and structure-activity relationships. Curr. Med. Chem 2005; 12:2601-23.

4. Halliwell B. Oxidative stress and neurodegeneration: where are we now? J. Neurochem 2006; 97:1634-58.

5. Shahidi F, Wanasundara PKJPD. Phenolic antioxidants. Critical Rev. in Food Sc. and Nutr 1992; 32:67-103.

6. Foti MC. Antioxidant properties of phenols. J of Pharm. and Pharmaco 2007: 59:1673-85.

7. Collier HOJ. The story of aspirin. In: Discoveries in Pharmacology. Ed Parnham MJ and Bruinvels J, editors. Elseviever New York 1987.

8. Soni MG, Burdocsk GA, Taylor SL, Greenberg NA. Safety assessment of propyl paraben: A review of the published literature. Food Chem. Tox 2001; 39:513-32.

9. Soni MG, Carabin IG, Burdocsk GA. Safety assessment of esters of p-hydroxybenzoic acid (parabens). Food Chem. Tox 2005; 43:985-1015.

10. Soni MG, Taylor SL, Greenberg NA, Burdocsk GA. Evaluation of the health aspects of methyl paraben: A review of the published literature. Food Chem. Tox 2002; 40:1335-73

11. Rice-Evans CA, Miller NJ, Paganga G. Structure - antioxidant activity relationship of flavonoids and phenolic acids. Free Radic. Biol. Med 1996; 20:933-56

12. Beauchamp C, Fridovich I. Superoxide dismutase: improved assays and an assay applicable to acrylamide gels. Anal. Biochem 1971; 44:276-87.

13. Dziedzic SZ, Hudson BJF. Polyhydroxychalconec and flavanones as antioxidant for edible oils. Food Chem 1983; 12:205-12.

14. Hansch C, Leo A, Taft RW. (1991)A survey of Hammett substituent constants and resonance and field parameters. Chem. Rev 1991; 91:165-95. 